\title{
The model-theoretic approach to aggregation: Impossibility results for finite and infinite electorates*
}

\author{
Frederik Herzberg ${ }^{\ddagger} \quad$ Daniel Eckert ${ }^{\S}$ \\ Copyright notice: The final publication, in Mathematical Social Sciences 64 \\ (2012), no. 1, pp. 41-47, is available at sciencedirect.com, \\ doi:10.1016/j.mathsocsci.2011.08.004
}

\begin{abstract}
It is well known that the literature on judgment aggregation inherits the impossibility results from the aggregation of preferences that it generalises. This is due to the fact that the typical judgment aggregation problem induces an ultrafilter on the the set of individuals. We propose a model-theoretic framework for the analysis of judgment aggregation and show that the conditions typically imposed on aggregators induce an ultrafilter on the set of individuals, thus establishing a generalised version of the Kirman-Sondermann correspondence. In the finite case, dictatorship then immediately follows from the principality of an ultrafilter on a finite set. This is not the case for an infinite set of individuals, where there exist free ultrafilters, as Fishburn already stressed in 1970. Following Lauwers and Van Liedekerke's (1995) seminal paper, we investigate another source of impossibility results for free ultrafilters: The domain of an ultraproduct over a free ultrafilter extends the individual factor domains, such that the preservation of the truth value of some sentences by the aggregate model - if this is as usual to be restricted to the original domain - may again require the exclusion of free ultrafilters, leading to dictatorship once again.

Key words: Arrow-type preference aggregation; judgment aggregation; model theory; first-order predicate logic; filter; ultrafilter; reduced product; ultraproduct; existential quantifier

2010 Mathematics Subject Classification: 91B14; 03C20; 03C98

Journal of Economic Literature classification: D71
\end{abstract}

\footnotetext{
*This work was partially supported by a German Research Foundation (DFG) grant. We are very grateful to an anonymous referee whose many valuable comments have been an enormous help in improving an earlier version of this paper.

${ }^{\dagger}$ Department of Mathematics, Princeton University, Fine Hall — Washington Road, Princeton, New Jersey 08544-1000, United States of America. fherzberg@math.princeton.edu

${ }^{\ddagger}$ Institut für Mathematische Wirtschaftsforschung, Universität Bielefeld, Universitätsstraße 25, D-33615 Bielefeld, Germany. fherzberg@uni-bielefeld.de

§Institut für Finanzwissenschaft, Karl-Franzens-Universität Graz, Universitätsstraße 15/E4, A-8010 Graz, Austria. daniel.eckert@uni-graz.at
} 


\section{Introduction}

In the last decades, the literature on social choice theory has seen important generalisations of the classical Arrovian problem of preference aggregation, starting with isolated contributions on abstract and algebraic aggregation theory by Wilson [16] resp. by Rubinstein and Fishburn [15] and culminating in the new field of judgment aggregation (for a survey see List and Puppe [12]). An essential feature of these generalisations is the extension of the problem of aggregation from the aggregation of preferences to the aggregation of arbitrary information. It thus seems natural to exploit the potential of model theory which, broadly speaking, studies the relation between abstract structures and statements about them (for an introduction to model theory see Bell and Slomson [1]) and to analyse the problem of judgment aggregation as the problem of aggregating the models that satisfy these judgments (section 2). This approach is justified by the fact that one of the major tools of model theory, namely the ultraproduct construction, can be shown to be equivalent to the construction of an aggregation rule satisfying properties in the spirit of the conditions of Arrow's impossibility theorem, an equivalence which is based on the role of ultrafilters in both cases. Thus a generalisation of the Kirman-Sondermann [9] correspondence between Arrovian aggregation rules and ultrafilters on the set of individuals is presented in section 3. For the case of a finite set of individuals, this equivalence immediately allows one to derive a dictatorship result, as ultrafilters on finite sets are necessary principal, whence the ultrafilter on a finite set of individuals always is the set of all supersets of a singleton - the dictator.

Whilst this dictatorship result does not carry over to the case of an infinite set of individuals (where free ultrafilters exist), we explore in section 4 another source of impossibility in the infinite case which was first identified by Lauwers and Van Liedekerke [10]. (Similar results for infinite populations are going to appear in another proceedings volume, cf. Herzberg and Eckert [7].) We conclude in section 5 by challenging the seemingly natural condition that an aggregate model has the same domain as the individual models.

\section{A model-theoretic framework for abstract aggregation theory}

Fix an arbitrary set $A$, and let $\mathcal{L}$ be a language consisting of constant symbols $\dot{a}$ for all elements $a$ of $A$ as well as (at most countably many) predicate symbols $\dot{P}_{n}, n \in \mathbf{N}$. We shall denote the arity of $\dot{P}_{n}$ by $\delta(n)$ (for all $n \in \mathbf{N}$ ) ${ }^{1}$

Let $\mathcal{S}$ be the set of atomic formulae in $\mathcal{L}$, and let $\mathcal{T}$ be the Boolean closure of $\mathcal{S}$, i.e. the closure of $\mathcal{S}$ under the logical connectives $\dot{\neg}, \dot{\wedge}, \dot{V}$.

Let $T$ be a consistent set of sentences in $\mathcal{L}$; we shall later impose additional assumptions on $T$ that are satisfied for every consistent universal $T{ }^{2}$

\footnotetext{
${ }^{1}$ We follow the common practise in mathematical logic of using dots on top of symbols to distinguish between symbols in the meta-language and those in the object language: Symbols of the object language will usually be dotted, whereas symbols of the meta-language will not. Note that a free variable which is to be interpreted as formula in the object language is still a symbol of the meta language and thus will not be dotted.

${ }^{2} \mathrm{~A}$ sentence is universal if it (in its prenex normal form) has the form $\left(\dot{\forall} \dot{v}_{k_{1}}\right) \cdots\left(\dot{\forall} \dot{v}_{k_{m}}\right) \dot{\psi}$
} 
The relational structure $\mathfrak{B}=\left\langle B,\left\{R_{n}: n \in \mathbf{N}\right\}\right\rangle$ with $A \subseteq B$ is called a realisation of $\mathcal{L}$ with domain $B$ or an $\mathcal{L}$-structure if and only if the arities of the relations $R_{n}$ correspond to the arities of the predicate symbols $\dot{P}_{n}$, that is if $R_{n} \subseteq B^{\delta(n)}$ for each $n$. The interpretation of the constant symbols does not need to be specified, but will be fixed uniformly for all $\mathcal{L}$-structures: For each $\mathcal{L}$-structure $\mathfrak{B}$, the interpretation of the constant symbol $\dot{a}$ is, for every $a \in A$, just $\dot{a}^{\mathfrak{B}}=a$. In other words, in this article, all $\mathcal{L}$-structures are understood to have a domain $\supseteq A$ and to interpret the constant symbols canonically (i.e. $\dot{a}$ is always interpreted by $a$, for all $a \in A$ ).

An $\mathcal{L}$-structure $\mathfrak{B}$ is a model of the theory $T$ if $\mathfrak{B} \models \varphi$ for all $\varphi \in T$, i.e. if all sentences of the theory hold true in $\mathfrak{B}$ (with the usual Tarski definition of $\operatorname{truth}^{3}$.

Let $\mathfrak{B}=\left\langle B,\left\{R_{n}: n \in \mathbf{N}\right\}\right\rangle$ be an $\mathcal{L}$-structure with domain $B$. (Note that this entails $A \subseteq B$ by our convention.) According to standard model-theoretic termininology (cf. e.g. Bell and Slomson [1, p. 73]), the restriction of $\mathfrak{B}$ to $A$ is the $\mathcal{L}$-structure $\left\langle A,\left\{R_{n} \cap A^{\delta(n)}: n \in \mathbf{N}\right\}\right\rangle$ and will be denoted by $\operatorname{res}_{A} \mathfrak{B}$. (In other words, the restriction of $\mathfrak{B}$ to $A$ is the $\mathcal{L}$-structure that is obtained by restricting the interpretations of the relation symbols to the domain $A \subseteq B$.)

Suppose now that $\mathfrak{B}=\left\langle B,\left\{R_{n}: n \in \mathbf{N}\right\}\right\rangle$ is a relational structure with $R_{n} \subseteq B^{\delta(n)}$ for each $n$ and such that there exists an injective map $i$ : $A \rightarrow B$. Then, the restriction of $\mathfrak{B}$ to $A$ under $i$ is the $\mathcal{L}$-structure $\left\langle A,\left\{i^{-1}\left[R_{n} \cap i[A]^{\delta(n)}\right]: n \in \mathbf{N}\right\}\right\rangle$ and will be denoted by $\operatorname{res}_{i, A} \mathfrak{B}$. We shall drop the subscript $i$ where no ambiguity can arise, e.g. if $A \subseteq B$ and $i$ is the identity map or if $\mathfrak{B}$ is the reduced product of $\mathfrak{A}$ with respect to some filter $\mathcal{D}$ and $i$ is the canonical embedding (see below for a definition of the reduced product) 4

Let $\Omega$ be the collection of models of $T$ with domain $A$. We assume that the following propositions hold for all $\mathcal{L}$-structures $\mathfrak{A}, \mathfrak{A}_{1}, \mathfrak{A}_{2}$ :

$$
\begin{gathered}
\left(\forall \lambda \in \mathcal{S} \quad\left(\mathfrak{A}_{1} \models \lambda \Leftrightarrow \mathfrak{A}_{2}=\lambda\right)\right) \Rightarrow \mathfrak{A}_{1}=\mathfrak{A}_{2} . \\
\mathfrak{A} \models T \Rightarrow \operatorname{res}_{A} \mathfrak{A} \in \Omega \\
\forall \lambda \in \mathcal{T} \quad\left(\mathfrak{A} \models \lambda \Leftrightarrow \operatorname{res}_{A} \mathfrak{A} \models \lambda\right) .
\end{gathered}
$$

Remark 2.1. If $\mathcal{S}$ is the set of all atomic $\mathcal{L}$-sentences, then the implication (1) holds for all $\mathfrak{A}_{1}, \mathfrak{A}_{2}$ with domain $A$.

Proof by contraposition. If $\mathfrak{A}_{1} \neq \mathfrak{A}_{2}$ for $\mathfrak{A}_{1}=\left\langle A,\left\{R_{n}^{1}: n \in \mathbf{N}\right\}\right\rangle$ and $\mathfrak{A}_{2}=$ $\left\langle A,\left\{R_{n}^{2}: n \in \mathbf{N}\right\}\right\rangle$, then $R_{n}^{1} \neq R_{n}^{2}$ for some $n \in \mathbf{N}$. Since $\mathfrak{A}_{1}, \mathfrak{A}_{2} \in \Omega$, both $R_{n}^{1}$ and $R_{n}^{2}$ are (different) subsets of $A^{\delta(n)}$. Hence, there exists some

\footnotetext{
for some formula $\psi$ that does not contain any quantifiers. A set of sentences is said to be universal if and only if all its elements are universal.

${ }^{3}$ For instance, if $\mathfrak{B}=\left\langle B,\left\{R_{n}: n \in \mathbf{N}\right\}\right\rangle$ is an $\mathcal{L}$-structure, then for all $a_{1}, \ldots, a_{\delta(n)} \in A$, one has

$$
\mathfrak{B} \models \dot{P}_{n}\left(\dot{a}_{1}, \ldots, \dot{a}_{\delta(n)}\right) \Leftrightarrow\left\langle a_{1}, \ldots, a_{\delta(n)}\right\rangle \in R_{n} .
$$

${ }^{4}$ One could also define the restriction of $\mathfrak{B}$ to $A$ as follows: Suppose $\mathfrak{A}$ and $\mathfrak{B}$ are $\mathcal{L}$ structures where the domain $A$ of $\mathfrak{A}$ is a subset of the domain $B$. If the inclusion mapping $i$ is an elementary embedding, then $\mathfrak{A}$ is the restriction of $\mathfrak{B}$ to $A$ and will be denoted $\operatorname{res} A^{\mathfrak{B}}$. This alternative definition is more general since it can also be used where $\mathcal{L}$-structures are allowed to have different, non-canonical interpretations for the constant symbols $\dot{a}, a \in A$ (which in our framework is excluded by definition).
} 
$\left\langle a_{1}, \ldots, a_{\delta(n)}\right\rangle \in A^{\delta(n)}$ such that either $\left\langle a_{1}, \ldots, a_{\delta(n)}\right\rangle \in R_{n}^{1}$ and $\left\langle a_{1}, \ldots, a_{\delta(n)}\right\rangle \notin$ $R_{n}^{2}$ or $\left\langle a_{1}, \ldots, a_{\delta(n)}\right\rangle \notin R_{n}^{1}$ and $\left\langle a_{1}, \ldots, a_{\delta(n)}\right\rangle \in R^{2}$. In both cases

$$
\left\langle a_{1}, \ldots, a_{\delta(n)}\right\rangle \in R_{n}^{1} \nLeftarrow\left\langle a_{1}, \ldots, a_{\delta(n)}\right\rangle \notin R_{n}^{2}
$$

hence

$$
\mathfrak{A}_{1} \models \dot{P}_{n}\left(\dot{a}_{1}, \ldots, \dot{a}_{\delta(n)}\right) \nLeftarrow \mathfrak{A}_{2} \models \dot{P}_{n}\left(\dot{a}_{1}, \ldots, \dot{a}_{\delta(n)}\right),
$$

although $\dot{P}_{n}\left(\dot{a}_{1}, \ldots, \dot{a}_{\delta(n)}\right) \in \mathcal{S}$.

Remark 2.2. If $T$ is universal, then the implication (2) holds for all $\mathcal{L}$ structures $\mathfrak{A}$.

Proof. If $T$ is universal and $\mathfrak{A} \models T$, then $\operatorname{res}_{A} \mathfrak{A} \models T$ and thus $\operatorname{res}_{A} \mathfrak{A} \in \Omega$.

Remark 2.3. If $\mathcal{S}$ is a set of atomic sentences, then the equivalence (3) holds for any $\mathcal{L}$-structure $\mathfrak{A}$.

Proof. If $\mathcal{S}$ only consists of atomic sentences, clearly

$$
\forall \lambda \in \mathcal{S} \quad\left(\mathfrak{A} \models \lambda \Leftrightarrow \operatorname{res}_{A} \mathfrak{A}=\lambda\right) .
$$

Since $\mathcal{T}$ is just the Boolean closure of $\mathcal{S}$, the equivalence $\sqrt{3}$ is established.

Let $I$ be a (finite or infinite) set. Elements of $I$ will be called individuals, elements of $\Omega^{I}$ will be called profiles. An aggregator is a map $f: \operatorname{dom}(f) \rightarrow$ $\operatorname{ran}(f)$ whose domain $\operatorname{dom}(f)$ is a subset of $\Omega^{I}$ and whose range $\operatorname{ran}(f)$ is a subset of $\Omega$.

This framework is sufficiently general to cover the cases of preference aggregation, propositional judgment aggregation, and modal aggregation:

- For the case of preference aggregation, the centrality of binary relations makes it particularly natural to express preferences by a binary predicate in first order logic (cf. Rubinstein [14, Lauwers and Van Liedekerke [10]). A more elaborate formalisation and complete axiomatisation of Arrow's theorem in first order logic was recently given by Grandi and Endriss [6].

- For propositional judgment aggregation à la Dietrich and List [3], one lets $\mathcal{L}$ have a single unary predicate $\dot{B}$, modelling a belief operator. The set $A$ will be the agenda. The interpretation of $\dot{B} \dot{a}$ is " $a$ is accepted". (Thus, the interpretation of $\mathfrak{A}_{i}=\dot{B} \dot{a}$ is "under profile $\underline{\mathfrak{A}}$, individual $i$ accepts $a$ ", and the interpretation of $f(\underline{\mathfrak{A}})=\dot{B} \dot{a}$ is "under profile $\underline{\mathfrak{A}}, a$ is socially accepted".) $T$ can be any universal theory in that language. (The use of the belief operator $B$ enables us to distinguish between sentences concerning acceptance or rejection of propositions in the agenda on the one hand and elements of the agenda themselves on the other hand. By that means we can take into account the more recent contributions on judgment aggregation without full rationality, cf. e.g. Dietrich and List [3], and allow judgment sets to be incomplete 5

\footnotetext{
${ }^{5}$ Put more simply: If the individuals are not assumed to have complete judgment sets, it makes a difference whether an individual actually rejects a proposition, i.e. accepts its negation, or simply does not endorse that proposition. If one wants to make this distinction in a formal framework, one needs a belief operator such as our $B$. We are grateful to an anonymous referee who helped us to formulate this point more clearly.
} 
- For modal propositional judgment aggregation, one simply uses the reduction of modal logic to first-order predicate logic, where the individuals correspond to possible states of the world. Thus, the set $A$ will be the set of states of the world. Let there be in $\mathcal{L}$ one predicate $M_{p}$ each for the elements $p$ of the agenda, modelling a modal belief operator with world argument and proposition index. Let there also be a binary predicate $\dot{R}$ in $\mathcal{L}$, denoting the accessibility relation. The interpretation of $\dot{R}(\dot{a}, \dot{b})$ will thus be " $b$ is accessible from world $a$ ". The interpretation of $\dot{M}_{p} \dot{a}$ will be "proposition $p$ is accepted in world $a$ ". (The interpretation of $\mathfrak{A}_{i}=\dot{M}_{p} \dot{a}$ is thus "under profile $\underline{\mathfrak{A}}$, individual $i$ accepts $p$ in world $a$ ", and the interpretation of $f(\underline{\mathfrak{A}}) \models \dot{M}_{p} \dot{\dot{a}}$ is "under profile $\underline{\mathfrak{A}}, p$ is socially accepted in world $a$ ".) The modal operator $\square$ will then not be an operator in the strict sense any longer, but in can be defined as a family of sentences, indexed by $p$ :

$$
\left.\square_{p} \dot{v}_{0}: \equiv \dot{(} \dot{\forall} \dot{v}_{1} \ddot{)} \dot{R}\left(\dot{v}_{0}, \dot{v}_{1}\right) \rightarrow \dot{M}_{p} \dot{v}_{1}\right)
$$

The interpretation of $\square_{p} \dot{a}$ is " $p$ is accepted in all worlds which are accessible from world $a$ ", or just " $p$ is necessarily accepted in world $a$ ". $T$ can be any universal theory in that language, which includes the axioms of the modal logical system employed (such as K, S4, S5, etc.).

\section{Kirman-Sondermann generalised and the impossibility in the finite case}

As the literature on judgment aggregation has amply shown, the interplay of the logical richness of the theory with the properties of aggregation rules is the driving force of the impossibility results. The reason for this lies in the fact that an important axiom for aggregation rules is the independence property, which requires that the social acceptance of any given sentence only depends on the individual support for this particular sentence (cf. Dietrich and List 4]).

For all $\lambda \in \mathcal{T}$ and all $\underline{\mathfrak{A}} \in \Omega^{I}$, we denote the coalition supporting $\lambda$ given profile $\underline{\mathfrak{A}}$, by

$$
C(\underline{\mathfrak{A}}, \lambda):=\left\{i \in I: \mathfrak{A}_{i} \models \lambda\right\} .
$$

An aggregator $f$ is said to be independent if and only if for all $\lambda \in \mathcal{T}$ and all $\underline{\mathfrak{A}}, \underline{\mathfrak{A}}^{\prime} \in \operatorname{dom}(f)$, such that $C(\underline{\mathfrak{A}}, \lambda)=C\left(\underline{\mathfrak{A}}^{\prime}, \lambda\right)$ one has $f(\underline{\mathfrak{A}}) \models \lambda$ if and only if $f\left(\overline{\mathfrak{A}}^{\prime}\right) \models \lambda$. by

Now, every aggregator $f$ induces a collection of decisive coalitions $\mathcal{C}_{f}^{\lambda}$ defined

$$
\mathcal{C}_{f}^{\lambda}:=\{C \subseteq I \quad: \forall \underline{\mathfrak{A}} \in \operatorname{dom}(f) \quad(C=C(\underline{\mathfrak{A}}, \lambda) \Rightarrow f(\underline{\mathfrak{A}}) \models \lambda)\},
$$

and it is easily seen that if $f$ is independent, then

$$
\begin{aligned}
\mathcal{C}_{f}^{\lambda} & =\{C \subseteq I: \exists \underline{\mathfrak{A}} \in \operatorname{dom}(f) \quad(C=C(\underline{\mathfrak{A}}, \lambda), \quad f(\underline{\mathfrak{A}}) \models \lambda)\} \\
& =\{C(\underline{\mathfrak{A}}, \lambda): \underline{\mathfrak{A}} \in \operatorname{dom}(f), \quad f(\underline{\mathfrak{A}}) \models \lambda\}
\end{aligned}
$$

We say that an aggregator $f$ is paretian if and only if $I \in \mathcal{C}_{f}^{\lambda}$ for all $\lambda \in \mathcal{T}$. The independence and the Pareto properties have considerable strength together which is well known from the contagion lemma in the theory of preference 
aggregation (according to which a decisive coalition for a particular pair of alternatives is decisive for any pair of alternatives) ${ }^{6}$

Consider any formulae $\varsigma, \tau \in \mathcal{T}$. Following standard terminology in the literature on judgment aggregation (see List and Puppe [12]), we say that $\varsigma$ conditionally entails $\tau$ under $T$ (written $\varsigma \vdash_{T}^{*} \tau$ ) if there exists $\lambda \in \mathcal{T}$ such that $\varsigma \dot{\wedge} \lambda \wedge \dot{\neg} \tau$ is inconsistent with $T$, while both $\varsigma \dot{\wedge} \lambda$ and $\lambda \dot{\lambda} \dot{\neg} \tau$ are consistent with $T$. The set $\mathcal{T}$ is said to be totally blocked if the transitive closure of the conditional entailment relation $\vdash_{T}^{*}$ is strongly connected, i.e. if any formula is related to any other one by a sequence of conditional entailments.

Lemma 3.1 (Contagion Lemma). Let $f: \Omega^{I} \rightarrow \Omega$ be paretian and independent. If $\mathcal{T}$ is totally blocked, then $\mathcal{C}_{f}^{\varsigma}=\mathcal{C}_{f}^{\tau}$ for all $\varsigma, \tau \in \mathcal{T}$.

Proof. Consider two sentences $\varsigma, \tau$ such that $\varsigma \vdash_{T}^{*} \tau$. Then there exists some $\lambda \in \mathcal{T}$ such that $T \cup\{\lambda, \varsigma\} \vdash \tau$. Given any independent and paretian aggregator $f$ as well as some $C \in \mathcal{C}_{f}^{\varsigma}$, construct a profile $\mathfrak{A} \in \Omega^{I}$ such that

1. $C=C(\underline{\mathfrak{A}}, \varsigma \dot{\wedge} \lambda)$

2. $N \backslash C=C(\underline{\mathfrak{A}}, \lambda \dot{\wedge} \dot{\neg} \tau)$, whence in particular

3. $C(\underline{\mathfrak{A}}, \lambda)=I$

Since $f$ is paretian, property 3 implies that $C(\underline{\mathfrak{A}}, \lambda) \in \mathcal{C}_{f}^{\lambda}$, hence $f(\underline{\mathfrak{A}}) \models \lambda$, and since $f$ is independent, property 1 implies that $f(\underline{\mathfrak{A}}) \models \varsigma$. It follows that $f(\underline{\mathfrak{A}}) \models \tau$ (since $\mathfrak{A}_{i}=T$ for all $i \in I$ as $\underline{\mathfrak{A}} \in \Omega^{I}$, hence $f(\underline{\mathfrak{A}}) \models T$ by the Pareto property, and $T \cup\{\lambda, \sigma\} \vdash \tau)$. In other words, $C(\underline{\mathfrak{A}}, \tau) \in \mathcal{C}_{f}^{\tau}$. However, by property 3, we have

$$
C(\underline{\mathfrak{A}}, \lambda \dot{\wedge} \dot{\neg} \tau)=C(\underline{\mathfrak{A}}, \dot{\neg} \tau)=N \backslash C(\underline{\mathfrak{A}}, \tau),
$$

thus $C(\mathfrak{A}, \tau)=C$ by property 2 . Hence, we have actually shown that $C=$ $C(\underline{\mathfrak{A}}, \tau) \in \mathcal{C}_{f}^{\tau}$. Since this holds for an arbitrary $C \in \mathcal{C}_{f}^{\varsigma}$ and for all $\sigma, \tau$ with $\varsigma \vdash_{T}^{*} \tau$, it follows that $\mathcal{C}_{f}^{\varsigma} \subseteq \mathcal{C}_{f}^{\tau}$ for all $\varsigma, \tau \in \mathcal{T}$ such that $\varsigma \vdash_{T}^{*} \tau$. If $\mathcal{T}$ is totally blocked, one has

$$
\varsigma \vdash_{T}^{*} \phi_{1} \vdash_{T}^{*} \cdots \vdash_{T}^{*} \phi_{m} \vdash_{T}^{*} \tau
$$

and

$$
\tau \vdash_{T}^{*} \psi_{1} \vdash_{T}^{*} \cdots \vdash_{T}^{*} \psi_{n} \vdash_{T}^{*} \varsigma
$$

for certain $\phi_{1}, \ldots \phi_{m}, \psi_{1}, \ldots, \psi_{n} \in \mathcal{T}(m, n \geq 0)$, hence

$$
\mathcal{C}_{f}^{\varsigma} \subseteq \mathcal{C}_{f}^{\phi_{1}} \subseteq \cdots \subseteq \mathcal{C}_{f}^{\phi_{m}} \subseteq \mathcal{C}_{f}^{\tau},
$$

i.e. $\mathcal{C}_{f}^{\varsigma} \subseteq \mathcal{C}_{f}^{\tau}$, and

$$
\mathcal{C}_{f}^{\tau} \subseteq \mathcal{C}_{f}^{\psi_{1}} \subseteq \cdots \subseteq \mathcal{C}_{f}^{\psi_{n}} \subseteq \mathcal{C}_{f}^{\varsigma},
$$

i.e. $\mathcal{C}_{f}^{\tau} \subseteq \mathcal{C}_{f}^{\varsigma}$, thus $\mathcal{C}_{f}^{\tau}=\mathcal{C}_{f}^{\varsigma}$.

\footnotetext{
${ }^{6}$ For the role of the contagion lemma in judgment and preference aggregation see List and Polak [1].
} 
Consider the set of decisive coalitions

$$
\mathcal{D}_{f}:=\{C \subseteq I \quad: \forall \lambda \in \mathcal{T} \quad \forall \underline{\mathfrak{A}} \in \operatorname{dom}(f) \quad(C=C(\underline{\mathfrak{A}}, \lambda) \Rightarrow f(\underline{\mathfrak{A}}) \models \lambda)\} .
$$

If $\mathcal{T}$ is totally blocked, then the Contagion Lemma allows us to characterise any paretian independent aggregator with universal domain in a neutral way by a single collection $\mathcal{D}_{f}=\mathcal{C}_{f}^{\lambda}$ for all $\lambda \in \mathcal{T}$. The set of decisive coalitions then simply becomes

$$
\begin{aligned}
\mathcal{D}_{f} & =\left\{\begin{array}{lll}
C \subseteq I & : \forall \lambda \in \mathcal{T} & C \in \mathcal{C}_{f}^{\lambda}
\end{array}\right\} \\
& =\left\{C \subseteq I: \exists \lambda \in \mathcal{T} \quad C \in \mathcal{C}_{f}^{\lambda}\right\} \\
& =\{C \subseteq I: \exists \lambda \in \mathcal{T} \quad \exists \underline{\mathfrak{A}} \in \operatorname{dom}(f) \quad(C=C(\underline{\mathfrak{A}}, \lambda) \Rightarrow f(\underline{\mathfrak{A}}) \models \lambda)\} \\
& =\{C(\underline{\mathfrak{A}}, \lambda): \lambda \in \mathcal{T}, \quad \underline{\mathfrak{A}} \in \operatorname{dom}(f), \quad f(\underline{\mathfrak{A}}) \models \lambda\} .
\end{aligned}
$$

We now introduce in the model-theoretic framework a set of axioms that are related to the standard assumptions on aggregation rules in the literature both on preference and on judgment aggregation.

(A1). $\operatorname{dom}(f)=\Omega^{I}$.

(A1'). There exist models $\mathfrak{A}_{1}, \mathfrak{A}_{2}, \mathfrak{A}_{3} \in \Omega$ such that

1. $\mathfrak{A}_{1}=\mu \dot{\wedge} \nu, \mathfrak{A}_{2} \models \mu \dot{\wedge} \dot{\neg} \nu, \mathfrak{A}_{3} \models \dot{\neg} \mu \dot{\wedge} \nu$, and

2. $\left\{\mathfrak{A}_{1}, \mathfrak{A}_{2}, \mathfrak{A}_{3}\right\}^{I} \subseteq \operatorname{dom}(f)$.

(A2). For all $\underline{\mathfrak{A}} \in \operatorname{dom}(f)$ and all $\lambda \in \mathcal{T}$, if $f(\underline{\mathfrak{A}}) \models \lambda$, then $C(\underline{\mathfrak{A}}, \lambda) \neq \emptyset$.

(A3). For all $\mathfrak{A}, \mathfrak{A}^{\prime} \in \operatorname{dom}(f)$ and all $\lambda, \lambda^{\prime} \in \mathcal{T}$ such that $C(\underline{\mathfrak{A}}, \lambda)=C\left(\underline{\mathfrak{A}}^{\prime}, \lambda^{\prime}\right)$, one has $f(\underline{\mathfrak{A}}) \models \lambda$ if and only if $f\left(\underline{\mathfrak{A}}^{\prime}\right) \models \lambda^{\prime}$.

(A1) is the axiom of Universal Domain of which Axiom (A1') is a significant weakening. Indeed, it is not difficult to prove that axiom (A1) implies axiom $\left(\mathrm{A} 1^{\prime}\right)$ :

Remark 3.2. If there exist $\mu, \nu \in \mathcal{S}$ such that $\mu \dot{\wedge} \nu, \mu \dot{\wedge} \dot{\neg} \nu, \dot{\neg} \mu \dot{\wedge} \nu$ are each consistent with $T$, then there already exist three pairwise different models $\mathfrak{A}_{1}, \mathfrak{A}_{2}, \mathfrak{A}_{3} \in \Omega$ such that $\mathfrak{A}_{1} \models \mu \dot{\wedge} \nu, \mathfrak{A}_{2}=\mu \dot{\wedge} \dot{\neg}$, and $\mathfrak{A}_{3} \models \dot{\neg} \mu \dot{\wedge} \nu$.

Proof. Since each of the three test sentences $\mu \dot{\wedge} \nu, \mu \dot{\wedge} \dot{\neg} \nu, \dot{\neg} \mu \dot{\wedge} \nu$ is consistent with $T$, the completeness of predicate logic yields models $\mathfrak{B}_{1}, \mathfrak{B}_{2}, \mathfrak{B}_{3}$ such that $\mathfrak{B}_{1} \models$ $\left.T \cup\{\mu \dot{\wedge} \nu\}, \mathfrak{B}_{2}=T \cup\{\mu \dot{\wedge} \dot{\nu}\rangle\right\}$, and $\mathfrak{B}_{3}=T \cup\{\dot{\neg} \mu \dot{\wedge} \nu\}$. Define $\mathfrak{A}_{1}:=\operatorname{res}_{A} \mathfrak{B}_{1}$, $\mathfrak{A}_{2}:=\operatorname{res}_{A} \mathfrak{B}_{2}$, and $\mathfrak{A}_{3}:=\operatorname{res}_{A} \mathfrak{B}_{3}$. By implication $(2), \mathfrak{A}_{1}, \mathfrak{A}_{2}, \mathfrak{A}_{3} \in \Omega$, and by equivalence (3), we have $\mathfrak{A}_{1}=\mu \dot{\wedge} \nu, \mathfrak{A}_{2}=\mu \dot{\wedge} \dot{\lrcorner} \nu$, and $\mathfrak{A}_{3}=\dot{\lrcorner} \mu \dot{\wedge} \nu$. Finally, since the three test sentences are pairwise inconsistent, the three models $\mathfrak{A}_{1}, \mathfrak{A}_{2}, \mathfrak{A}_{3}$ must be pairwise different.

Axiom (A2) is a generalised Pareto Principle obtained by weakening the usual Pareto property. (A3) is a generalised form of the axiom of Systematicity, which itself is a combination of the independence property and the neutrality property implied by the Contagion Lemma. Admittedly, it is hard to find a direct justification for this strong condition and its relaxation plays an important role in the literature on judgment aggregation (see e.g. Pauly and van Hees [13]). 
However, the contagion of decisiveness via conditional entailment plays such a central role in aggregation problems that systematicity can be considered the reference case.

An aggregator $f$ will be called Arrow-rational if and only if the axioms (A1),(A2),(A3) are satisfied for $\mathcal{T} ; f$ will be called weakly Arrow-rational if and only if the axioms $\left(\mathrm{A} 1^{\prime}\right),(\mathrm{A} 2),(\mathrm{A} 3)$ are satisfied.

We denote by $\mathcal{A R}$ the set of all Arrow-rational aggregators, and by $\mathcal{A} \mathcal{R}^{\prime}$ the set of all weakly Arrow-rational aggregators. Since (A1) implies (A1') (see Remark $3.2, \mathcal{A R} \subseteq \mathcal{A} \mathcal{R}^{\prime}$.

Remark 3.3. If $f$ satisfies (A3), then for all $\underline{\mathfrak{A}} \in \operatorname{dom}(f)$ and $\lambda \in \mathcal{T}$,

$$
C(\underline{\mathfrak{A}}, \lambda) \in \mathcal{D}_{f} \Leftrightarrow f(\underline{\mathfrak{A}}) \models \lambda .
$$

Proof. Suppose $f$ satisfies (A3), let $\mathfrak{A} \in \operatorname{dom}(f)$ and $\lambda \in \mathcal{T}$. By definition, $f(\mathfrak{A})=\lambda$ implies $C(\mathfrak{A}, \lambda) \in \mathcal{D}_{f}$. Conversely, if $C(\mathfrak{A}, \lambda) \in \mathcal{D}_{f}$, then there exist $\underline{\mathfrak{A}}^{\prime} \in \operatorname{dom}(f)$ and $\lambda^{\prime} \in \mathcal{T}$ with $f\left(\underline{\mathfrak{A}}^{\prime}\right)=\lambda^{\prime}$ and $C(\underline{\mathfrak{A}}, \lambda)=C\left(\underline{\mathfrak{A}}^{\prime}, \lambda^{\prime}\right)$. As $f$ satisfies (A3), this means $f(\underline{\mathfrak{A}}) \models \lambda$.

Now we verify the (ultra)filter properties of $\mathcal{D}_{f}$ :

Definition 3.4. A collection $\mathcal{D} \subseteq 2^{I}$ of coalitions is a filter on I if and only if it has the following properties:

(F1). $\varnothing \notin \mathcal{D}$ (non-triviality).

(F2). For all $C \in \mathcal{D}$ and $C^{\prime} \in 2^{I}$, if $C \subseteq C^{\prime}$ then $C^{\prime} \in \mathcal{D}$ (closure under supersets).

(F3). For all $C, C^{\prime} \in \mathcal{D}, C \cap C^{\prime} \in \mathcal{D}$ (closure under intersections).

A filter $\mathcal{D} \subseteq 2^{I}$ is an ultrafilter if for all $C \in 2^{I}$ either $C \in \mathcal{D}$ or $I \backslash C \in \mathcal{D}$ (but not both).

Lemma 3.5. Let $f \in \mathcal{A} \mathcal{R}^{\prime}$, then $\mathcal{D}_{f}$ is an ultrafilter.

Proof. We have to verify the ultrafilter properties for $\mathcal{D}_{f}$. First, since $f$ satisfies $(\mathrm{A} 2), \mathcal{D}_{f}$ cannot contain $\emptyset$.

The next property which we have to verify is that $\mathcal{D}_{f}$ is closed under supersets; we will need this result in order to show that $\mathcal{D}_{f}$ is closed under intersections. Let, for that purpose, $C \in \mathcal{D}_{f}$ and $C^{\prime} \subseteq I$ with $C \subseteq C^{\prime}$. We shall show that $C^{\prime} \in \mathcal{D}_{f}$. Since $f$ satisfies (A1'), the domain of $f$ contains a profile $\underline{\mathfrak{A}}$ such that

$$
\begin{aligned}
& \forall i \in C \quad \mathfrak{A}_{i} \models \mu \dot{\wedge} \nu \\
& \forall j \in C^{\prime} \backslash C \quad \mathfrak{A}_{j} \models \mu \dot{\wedge} \dot{\neg} \nu \\
& \forall k \in I \backslash C^{\prime} \quad \mathfrak{A}_{k} \models \dot{\neg} \mu \dot{\wedge} \nu .
\end{aligned}
$$

Note that $C(\mathfrak{A}, \mu \dot{\wedge} \nu)=C \in \mathcal{D}_{f}$, so $f(\mathfrak{A}) \models \mu \dot{\wedge} \nu$ due to Remark 3.3 In particular, $f(\underline{\mathfrak{A}}) \models \mu$, whence readily $C(\underline{\mathfrak{A}}, \mu) \in \mathcal{D}_{f}$. On the other hand, however, $C^{\prime}=C(\underline{\mathfrak{A}}, \mu)$ by the choice of $\underline{\mathfrak{A}}$. Summarising this, we arrive at $C^{\prime} \in \mathcal{D}_{f}$. 
Now we prove that $\mathcal{D}_{f}$ is closed under intersections. Let $C, C^{\prime} \subseteq I$. Again since $f$ satisfies $\left(\mathrm{A}^{\prime}\right)$, there must be a profile $\underline{\mathfrak{A}} \in \operatorname{dom}(f)$ such that

$$
\begin{array}{rll}
\forall i \in C \cap C^{\prime} & \mathfrak{A}_{i} \models \mu \dot{\wedge} \nu \\
\forall j \in C \backslash\left(C \cap C^{\prime}\right) & \mathfrak{A}_{j} \models \mu \dot{\wedge} \dot{\sim} \nu \\
\forall k \in I \backslash C & \mathfrak{A}_{k} \models \dot{\sim} \mu \dot{\wedge} \nu .
\end{array}
$$

Then $C=C(\underline{\mathfrak{A}}, \mu) \in \mathcal{D}_{f}$, so $f(\underline{\mathfrak{A}}) \models \mu$ by Remark 3.3 . Also $C^{\prime} \subseteq C(\underline{\mathfrak{A}}, \nu)$ and $C^{\prime} \in \mathcal{D}_{f}$, therefore $C(\underline{A}, \nu) \in \mathcal{D}_{f}$, as we have already shown that $\overline{\mathcal{D}}_{f}$ is closed under supersets. Again by Remark 3.3, we obtain $f(\underline{\mathfrak{A}}) \models \nu$, too. Thus, $f(\underline{\mathfrak{A}}) \models \mu \dot{\wedge} \nu$, therefore $C \cap C^{\prime}=C(\underline{\mathfrak{A}}, \mu \dot{\wedge} \nu) \in \mathcal{D}_{f}$.

Finally, we need to show that $\mathcal{D}_{f}$ is an ultrafilter, not merely a filter. Hence, let $C \subseteq I$, and let us show that $C \in \mathcal{D}_{f}$ or $I \backslash C \in \mathcal{D}_{f}$. Since $f$ satisfies (A1'), the domain of $f$ contains a profile $\underline{\mathfrak{A}}$ such that

$$
\begin{aligned}
\forall i \in C & \mathfrak{A}_{i}=\mu \dot{\wedge} \dot{\neg} \nu \\
\forall j \in I \backslash C & \mathfrak{A}_{j} \equiv \dot{\neg} \mu \dot{\wedge} \nu .
\end{aligned}
$$

Then $\mathfrak{A}_{i}=\dot{(} \mu \dot{\vee} \nu \dot{)} \dot{\wedge} \dot{\neg}(\mu \dot{\wedge} \nu)$ for all $i \in I$, therefore $\mathfrak{A}_{i}=\dot{\neg}(\dot{(} \mu \dot{\vee} \nu \dot{\wedge} \dot{\wedge} \dot{\neg}(\mu \dot{\wedge} \nu))$ for no $i \in I$. In other words,

$$
C(\underline{\mathfrak{A}}, \dot{\neg} \ddot{(}(\mu \dot{\vee} \nu) \dot{\wedge} \dot{\wedge} \dot{\sim}(\mu \dot{\wedge} \nu) \ddot{)}))=\emptyset,
$$

whence

$$
f(\underline{\mathfrak{A}}) \forall=\dot{\neg}(\dot{(} \mu \dot{\vee} \nu \dot{)} \dot{\wedge} \dot{\neg}(\mu \dot{\wedge} \nu) \ddot{)}
$$

as $f$ satisfies (A2). Therefore,

$$
f(\underline{\mathfrak{A}}) \models \dot{(} \mu \dot{\vee} \nu \dot{)} \dot{\wedge} \dot{\neg}(\mu \dot{\wedge} \nu \dot{)} .
$$

This means that either $f(\underline{\mathfrak{A}}) \models \mu$ or $f(\underline{\mathfrak{A}}) \models \nu$, hence either $C(\underline{\mathfrak{A}}, \mu) \in \mathcal{D}_{f}$ or $I \backslash C=C(\underline{\mathfrak{A}}, \nu) \in \mathcal{D}_{f}$. However, $I \backslash C=C(\underline{\mathfrak{A}}, \nu)$ and $C=C(\underline{\mathfrak{A}}, \mu)$ by construction of $\underline{\mathfrak{A}}$. Hence, either $C \in \mathcal{D}_{f}$ or $I \backslash C \in \mathcal{D}_{f}$.

A well-known construction from model theory is based precisely on the notion of a (ultra)filter and captures the idea that what holds in "many" (measured by means of the (ultra)filter) individual structures should hold in the aggregate structure. This is the reduced product (or ultraproduct) construction.

In order to define this concept, let us fix a filter $\mathcal{D}$ on $I$. Define a relation $\sim_{\mathcal{D}}$ on $A^{I}$ by

$$
\forall \underline{a}, \underline{a}^{\prime} \in A^{I} \quad \underline{a} \sim_{\mathcal{D}} \underline{a}^{\prime} \Leftrightarrow\left\{i \in I \quad: a_{i}=a_{i}^{\prime}\right\} \in \mathcal{D} .
$$

The filter properties of $\mathcal{D}$ make it easy to verify that $\sim_{\mathcal{D}}$ is an equivalence relation. For any $\underline{a} \in A^{I}$, the equivalence class of $\underline{a}$ with respect to $\sim_{\mathcal{D}}$ shall be denoted $[\underline{a}]_{\mathcal{D}}$, and we shall write $A^{I} / \mathcal{D}$ for the set of all such equivalence classes.

Definition 3.6. Let $\mathcal{D}$ be a filter on $I$. Consider a family $\left\langle\mathfrak{A}_{i}\right\rangle_{i \in I}$ of $\mathcal{L}$ structures, and denote by $R_{n, i}$ the interpretation of predicate $\dot{P}_{n}$ under $\mathfrak{A}_{i}$. 
The reduced product of $\left\langle\mathfrak{A}_{i}\right\rangle_{i \in I}$ with respect to $\mathcal{D}$ is an $\mathcal{L}$-structure $\mathfrak{B}=$ $\left\langle B,\left\{R_{n}: n \in \mathbf{N}\right\}\right\rangle$ wherein $B=A^{I} / \mathcal{D}$ and

$$
R_{n}=\left\{\left\langle\left[\underline{a}^{1}\right]_{\mathcal{D}}, \ldots,\left[\underline{a}^{\delta(n)}\right]_{\mathcal{D}}\right\rangle:\left\{i \in I:\left\langle a_{i}^{1}, \ldots, a_{i}^{\delta(n)}\right\rangle \in R_{n . i}\right\} \in \mathcal{D}\right\}
$$

for all $n \in \mathbb{N}$.

The reduced product of $\left\langle\mathfrak{A}_{i}\right\rangle_{i \in I}$ with respect to $\mathcal{D}$ will be denoted $\prod_{i \in I} \mathfrak{A}_{i} / \mathcal{D}$. If $\mathcal{D}$ is an ultrafilter on $I$, then $\prod_{i \in I} \mathfrak{A}_{i} / \mathcal{D}$ is called the ultraproduct of $\left\langle\mathfrak{A}_{i}\right\rangle_{i \in I}$ with respect to $\mathcal{D}$.

Note that the filter properties of $\mathcal{D}$ ensure that the above definition of $R_{n}$, the interpretation of $\dot{P}_{n}$ under the reduced product, is well-defined!

Remark 3.7. Let $\mathcal{D}$ be a filter on $I$ and $\mathfrak{A} \in \Omega^{I}$. Then

$$
\operatorname{res}_{A} \prod_{i \in I} \mathfrak{A}_{i} / \mathcal{D} \models \lambda \Leftrightarrow C(\underline{\mathfrak{A}}, \lambda) \in \mathcal{D}
$$

for all $\lambda \in \mathcal{S}$. If $\mathcal{D}$ is an ultrafilter, then equivalence (4) holds even for all $\lambda \in \mathcal{T}$.

Proof. Let $\mathcal{D}$ be a filter, let $\underline{\mathfrak{A}} \in \Omega^{I}$ and let $\lambda \in \mathcal{T}$. By equivalence (3),

$$
\operatorname{res}_{A} \prod_{i \in I} \mathfrak{A}_{i} / \mathcal{D} \models \lambda \Leftrightarrow \prod_{i \in I} \mathfrak{A}_{i} / \mathcal{D} \models \lambda .
$$

Now, for all $\lambda \in \mathcal{S}$, one has

$$
\operatorname{res}_{A} \prod_{i \in I} \mathfrak{A}_{i} / \mathcal{D} \models \lambda \Leftrightarrow\left\{i \in I: \mathfrak{A}_{i}=\lambda\right\} \in \mathcal{D}
$$

by definition of the reduced product, which already is equivalence (4) (by the definition of $C(\underline{\mathfrak{A}}, \lambda)$ ). If $\mathcal{D}$ is an ultrafilter, then Łoś's theorem assures us that this equivalence even holds for all $\lambda \in \mathcal{T}$.

Lemma 3.8. Let $f \in \mathcal{A} \mathcal{R}^{\prime}$, then $f(\underline{\mathfrak{A}})=\operatorname{res}_{A} \prod_{i \in I} \mathfrak{A}_{i} / \mathcal{D}_{f}$ for all $\mathfrak{A} \in \operatorname{dom}(f)$.

Proof. By Lemma 3.5. $\mathcal{D}_{f}$ is a filter, whence $\operatorname{res}_{A} \prod_{i \in I} \mathfrak{A}_{i} / \mathcal{D}_{f}$ is well-defined for all $\mathfrak{A} \in \operatorname{dom}(f)$. Let us now fix arbitrary $\underline{\mathfrak{A}} \in \operatorname{dom}(f)$ and $\lambda \in \mathcal{S}$. Combining Remark 3.7 and Remark 3.3 ,

$$
\operatorname{res}_{A} \prod_{i \in I} \mathfrak{A}_{i} / \mathcal{D}_{f} \models \lambda \Leftrightarrow C(\underline{\mathfrak{A}}, \lambda) \in \mathcal{D}_{f} \Leftrightarrow f(\underline{\mathfrak{A}}) \models \lambda,
$$

thus $\operatorname{res}_{A} \prod_{i \in I} \mathfrak{A}_{i} / \mathcal{D}_{f} \models \lambda \Leftrightarrow f(\underline{\mathfrak{A}}) \models \lambda$. Since $\lambda \in \mathcal{S}$ was arbitrary, we deduce by means of implication (1) that $f(\underline{\mathfrak{A}})=\operatorname{res}_{A} \prod_{i \in I} \mathfrak{A}_{i} / \mathcal{D}_{f}$.

Lemma 3.9. Suppose $\mathcal{D}$ is an ultrafilter, and consider the aggregator $\operatorname{res}_{A} \prod / \mathcal{D}$, defined by

$$
\operatorname{res}_{A} \prod / \mathcal{D}: \Omega^{I} \rightarrow \Omega, \quad \underline{\mathfrak{A}} \mapsto \operatorname{res}_{A} \prod_{i \in I} \mathfrak{A}_{i} / \mathcal{D} .
$$

Then $\operatorname{res}_{A} \prod / \mathcal{D} \in \mathcal{A R}$. 
Proof. Let $\underline{\mathfrak{A}} \in \Omega^{I}$. Clearly, both the ultraproduct $\prod_{i \in I} \mathfrak{A}_{i} / \mathcal{D}$ and its restriction to $A$ are well-defined. By Łoś's theorem, $\prod_{i \in I} \mathfrak{A}_{i} / \mathcal{D} \models T$, and by implication (2), $\operatorname{res}_{A} \prod_{i \in I} \mathfrak{A}_{i} / \mathcal{D} \in \Omega$. Therefore, $\operatorname{res}_{A} \prod / \mathcal{D}: \Omega^{I} \rightarrow \Omega$. Let us now verify the axioms (A1), (A2), and (A3) for $\operatorname{res}_{A} \prod / \mathcal{D}$.

(A1). As we have just seen, $\operatorname{res}_{A} \Pi / \mathcal{D}$ is well-defined on $\Omega^{I}$.

(A2). Let $\underline{\mathfrak{A}} \in \Omega^{I}$ and $\lambda \in \mathcal{T}$ such that $\operatorname{res}_{A} \prod / \mathcal{D}(\underline{\mathfrak{A}}) \models \lambda$, that is $\operatorname{res}_{A} \bar{\prod}_{i \in I} \mathfrak{A}_{i} / \mathcal{D} \models \lambda$. Then, by Remark 3.7, we have $C(\underline{\mathfrak{A}}, \lambda) \in \mathcal{D}$, hence $C(\underline{\mathfrak{A}}, \lambda) \neq \emptyset$ as $\mathcal{D}$ is an ultrafilter.

(A3). For all $\underline{\mathfrak{A}}, \underline{\mathfrak{A}}^{\prime} \in \Omega^{I}$ and all $\lambda, \lambda^{\prime} \in \mathcal{T}$ such that $C(\underline{\mathfrak{A}}, \lambda)=C\left(\underline{\mathfrak{A}}^{\prime}, \lambda^{\prime}\right)$, Remark 3.7 entails that $\operatorname{res}_{A} \Pi / \mathcal{D}(\underline{\mathfrak{A}}) \models \lambda$ if and only if $\operatorname{res}_{A} \prod / \mathcal{D}\left(\underline{\mathfrak{A}}^{\prime}\right) \models \lambda^{\prime}$.

Let $\beta I$ denote the set of all ultrafilters on the set $I$.

Theorem 3.10 (Kirman-Sondermann correspondence (generalised)). There is a bijection between $\mathcal{A R}$ and $\beta I$, given by $\Lambda: \mathcal{A R} \rightarrow \beta I, \quad f \mapsto \mathcal{D}_{f}$. Its inverse is given by $\Lambda^{-1}: \beta I \rightarrow \mathcal{A R}, \mathcal{D} \mapsto \operatorname{res}_{A} \prod / \mathcal{D}$, wherein, as in Lemma 3.9. $\operatorname{res}_{A} \prod / \mathcal{D}$ denotes the aggregator $\underline{\mathfrak{A}} \mapsto \operatorname{res}_{A} \prod_{i \in I} \mathfrak{A}_{i} / \mathcal{D}$.

Proof. First, note that for all $f \in \mathcal{A R}, \Lambda(f)=\mathcal{D}_{f}$ is an ultrafilter by Lemma 3.5 . whence the range of $\Lambda$ is indeed a subset of $\beta I$. Next, it is easy to see that the map $\mathcal{D} \mapsto \operatorname{res}_{A} \prod / \mathcal{D}$ must be inverse to $\Lambda$ because Lemma 3.8 teaches that $f=\operatorname{res}_{A} \prod / \mathcal{D}_{f}$, in other words $\operatorname{res}_{A} \Pi /(\Lambda(f))=f$ for all $f \in \mathcal{A R}$.

Furthermore, since $\Lambda$ has an inverse $\Lambda^{-1}$, it must be injective. (Indeed, if $\Lambda(f)=\Lambda(g)$ for any $f, g \in \mathcal{A R}$, then $f=\Lambda^{-1}(\Lambda(f))=\Lambda^{-1}(\Lambda(g))=g$.) Finally, by Lemma 3.9 , the range of $\Lambda^{-1}$ is contained in $\mathcal{A R}$. Hence, for any $\mathcal{D} \in \beta I$, the aggregator $\Lambda^{-1}(\mathcal{D})$ is in the domain of $\Lambda$, whence $\mathcal{D}=\Lambda\left(\Lambda^{-1}(\mathcal{D})\right)$ is in the range of $\Lambda$. Therefore, $\Lambda$ is surjective.

As corollary of this result we immediately obtain the well-known impossibility result for a finite set of individuals.

We say that $f$ is dictatorial if and only if there exists some $i_{f} \in I$ such that $\mathcal{D}_{f}=\left\{J \subseteq I: i_{f} \in J\right\}$.

Remark 3.11. Let $f$ be an aggregator, and suppose $I$ is finite. Then, $f$ is dictatorial if and only if $\mathcal{D}_{f}$ is an ultrafilter.

Proof. A filter on a finite set is an ultrafilter if and only if its intersection is a singleton. Thus, $\left\{i_{f}\right\}=\bigcap \mathcal{D}_{f}$.

Corollary 3.12 (Impossibility theorem). Suppose I is finite. If $f \in \mathcal{A} \mathcal{R}$, then $f$ is dictatorial.

\section{Impossibility theorems for infinite populations}

For the following, assume that there is some filter $\mathcal{D}_{f}$ on $I$ such that for all $\underline{\mathfrak{A}} \in \operatorname{dom}(f)$,

$$
f(\underline{\mathfrak{A}})=\operatorname{res}_{A} \prod_{i \in I} \mathfrak{A}_{i} / \mathcal{D}_{f} .
$$


Observe that the restriction to $A$ is important since it is a necessary condition (for $f$ to be an aggregator) that the aggregate model $f(\underline{\mathfrak{A}})$ belongs to $\Omega$ and thus must have $A$ as its domain. Moreover, if $\mathcal{D}_{f}$ is an ultrafilter, then, by application of Łos's theorem, for every $\mathcal{L}$-sentence $\psi$,

$$
\prod_{i \in I} \mathfrak{A}_{i} / \mathcal{D}_{f} \mid=\psi \Leftrightarrow\left\{i \in I: \mathfrak{A}_{i} \models \psi\right\} \in \mathcal{D}_{f},
$$

which guarantees that $\prod_{i \in I} \mathfrak{A}_{i} / \mathcal{D}_{f} \models T$ and hence $f(\underline{\mathfrak{A}})=\operatorname{res}_{A} \prod_{i \in I} \mathfrak{A}_{i} / \mathcal{D}_{f} \models$ $T$ since $T$ consists only of universal sentences. Therefore, if $f$ is given as the restriction of an ultraproduct to $A$, then $f(\underline{\mathfrak{A}}) \in \Omega$ for all profiles $\underline{\mathfrak{A}} \in \Omega^{I}$.

For the case of an infinite set of individuals there exist free ultrafilters and therefore Arrow's impossibility theorem does not apply (as was already shown by Fishburn [5]).

However, the very construction of an ultraproduct bears another source of impossibility results as remarked by Lauwers and Van Liedekerke [10]: Ultraproducts with respect to free ultrafilters have a strictly larger domain than the factor structures, and thus, witnesses to certain existential statements in the ultraproduct do not need to belong to the domain of the factor structures (cf. Hodges [8] for a more comprehensive discussion of the role of ultraproducts for the construction of extensions of given structures). Therefore, if an aggregator is the restriction (to the factor-domain) of an ultraproduct $7^{7}$ and is required to preserve some non-universal statement (for example: existence of a best alternative; continuity; etc.), it must be the restriction of an ultraproduct with respect to a principal ultrafilter and will thus be dictatorial.

Indeed, Lauwers and Van Liedekerke [10, have remarked that in the setting of preference aggregation, the preservation of non-universal formulae generically leads to dictatorial impossibility results. For example, the deeper reason for Campbell's 2 theorem — which asserts the impossibility of satisfying Arrow's rationality axioms plus non-dicatorship even in infinite populations when preferences are assumed to be continuous - is that the formula defining continuity of preference orderings is non-universal. (To be more precise, it is neither universal nor existential, not even a so-called $\forall \exists$ formula ${ }^{8}$. The same phenomenon can be observed in the more general setting of first-order predicate aggregation theory.

In order to illustrate this, let us consider the simplest case, viz. preservation of a formula with one existential quantifier in a restricted ultraproduct construction. Suppose hence $\psi=(\dot{\exists} \dot{v}) \phi(\dot{v})$ for some $\mathcal{L}$-formula $\phi(\dot{v})$ with one free variable, assume $I$ is infinite, let $\mathcal{D}$ be an ultrafilter on $I$, and consider a family $\mathfrak{A}=\left\langle\mathfrak{A}_{i}\right\rangle_{i \in I}$ of models of $T$, all with the same domain $A$. Suppose that whilst $\dot{(\exists} \dot{\xi} \dot{)} \phi(\dot{v})$ is true in all models $\mathfrak{A}_{i}$, there does not exist an almost uniform witness, i.e. there exists no $a \in A$ such that $\phi[\dot{a}]$ would be true in $\mathcal{D}$-almost all models $\mathfrak{A}_{i}$. Then, Łoś's theorem teaches that $\phi[\dot{a}]$ fails in $\prod_{i \in I} \mathfrak{A}_{i} / \mathcal{D}$ for all $a \in A$, and therefore $\psi$ cannot be true in the restriction of $\prod_{i \in I} \mathfrak{A}_{i} / \mathcal{D}$ to $A$.

\footnotetext{
${ }^{7}$ For instance, Arrovian preference aggregators always map every profile to the restriction - to the set of alternatives - of its ultraproduct with respect to the ultrafilter of decisive coalitions, cf. Lauwers and Van Liedekerke [10].

${ }^{8}$ These are formulae which, in their prenex normal form, start with a (possibly empty) block of universal quantifiers followed by a (possibly empty) block of existential quantifiers and finally a quantifier-free formula.) The class of $\forall \exists$ formulae is interesting from a modeltheoretic vantage point, as the class of models of any theory that consists of $\forall \exists$ formulae only is closed under forming unions of elementary chains (another model-theoretic limit construction).
} 
This phenomenon can be used as a source of more general impossibility theorems in abstract aggregation theory. Our result will give a sufficient condition for aggregators that preserve some non-universal sentence to be a dictatorship.

For this purpose, let us fix an arbitrary non-universal formula. In its prenex normal form it can then be written as $\psi \equiv \ddot{(\forall} \dot{x}_{1} \dot{)} \ldots\left(\dot{\forall} \dot{x}_{m} \dot{)} \dot{(} \dot{y} \dot{y}\right) \phi\left(\dot{x}_{1}, \ldots, \dot{x}_{m} ; \dot{y}\right)$, wherein $m$ is a nonnegative integer and $\phi\left(\dot{x}_{1}, \ldots, \dot{x}_{m} ; \dot{y}\right)$ is some $\mathcal{L}$-formula with $m+1$ free variables 9 For the rest of this paper, $\psi$ and $\phi$ are thus fixed.

In order to formulate our results, we need some more terminology. We say that a profile $\mathfrak{A} \in \Omega^{I}$ has finite witness multiplicity with respect to $\phi$ if and only if $\mathfrak{A}_{i} \models \bar{\psi}$ for all $i \in I$, but for all $a_{1}, \ldots, a_{m}, a^{\prime} \in A$, the coalition $\left\{i \in I: \mathfrak{A}_{i}=\phi\left(\dot{a}_{1}, \ldots, \dot{a}_{m} ; \dot{a}^{\prime}\right)\right\}$ is finite. An aggregator $f$ is said to preserve an $\mathcal{L}$-sentence $\psi$ if and only if for all $\underline{\mathfrak{A}} \in \operatorname{dom}(f)$, one has $f(\underline{\mathfrak{A}}) \models \psi$ whenever $\mathfrak{A}_{i}=\psi$ for all $i \in I$. Moreover, we say that $\phi$ is free of negation, disjunction and universal quantification if and only if its non-abbreviated form does not contain the symbols $\dot{\neg}, \dot{V}$ nor $\dot{\forall}$, in other words, if the only logical symbols appearing in it are $\dot{\wedge}$ and $\dot{\exists}$. Henceforth we assume $\phi$ to possess this property 0

We can now state the impossibility theorem announced previously:

Theorem 4.1. Let $f$ be an aggregator that preserves $\psi$, and assume that there exists some $\underline{\mathfrak{A}} \in \operatorname{dom}(f)$ with finite witness multiplicity with respect to $\phi$.

1. If $\mathcal{D}_{f}$ is an ultrafilter, then it is also principal (whence $f$ is a dictatorship).

2. $\mathcal{D}_{f}$ contains a finite coalition (whence $f$ is an oligarchy).

Proof of Theorem 4.1. 1. Since $f(\underline{\mathfrak{A}})$ is just the $A$-restriction of the ultraproduct of $\underline{\mathfrak{A}}$ with respect to $\mathcal{D}_{f}$, Łoś's theorem readily yields the equivalence

$$
\begin{aligned}
& f(\underline{\mathfrak{A}}) \models \phi\left(\dot{a}_{1}, \ldots, \dot{a}_{m} ; \dot{a}^{\prime}\right) \\
\Leftrightarrow \quad & \left\{i \in I: \mathfrak{A}_{i} \models \phi\left(\dot{a}_{1}, \ldots, \dot{a}_{m} ; \dot{a}^{\prime}\right)\right\} \in \mathcal{D}_{f}
\end{aligned}
$$

for all $a_{1}, \ldots, a_{m}, a^{\prime} \in A$. Since $\underline{\mathfrak{A}}$ is assumed to have finite witness multiplicity with respect to $\phi$, we know that $\left\{i \in I: \mathfrak{A}_{i} \models \phi\left(\dot{a}_{1}, \ldots, \dot{a}_{m} ; \dot{a}^{\prime}\right)\right\}$ is finite for all $a_{1}, \ldots, a_{m}, a^{\prime} \in A$, and that $\mathfrak{A}_{i} \models \psi$ for all $i \in I$, whence $f(\underline{\mathfrak{A}}) \models \psi$ as $f$ preserves $\psi$. Therefore, for all $a_{1}, \ldots, a_{m} \in A$ there is some $a^{\prime} \in A$ such that $f(\underline{\mathfrak{A}}) \models \phi\left[\dot{a}_{1}, \ldots, \dot{a}_{m} ; \dot{a}^{\prime}\right]$, hence

$$
\left\{i \in I: \mathfrak{A}_{i}=\phi\left[\dot{a}_{1}, \ldots, \dot{a}_{m} ; \dot{a}^{\prime}\right]\right\} \in \mathcal{D}_{f}
$$

by equivalence (5), although

$$
C_{\vec{a}, a^{\prime}}:=\left\{i \in I: \mathfrak{A}_{i} \models\left[\dot{a}_{1}, \ldots, \dot{a}_{m} ; \dot{a}^{\prime}\right]\right\}
$$

is finite. Thus, the ultrafilter $\mathcal{D}_{f}$ contains a finite subset of $I$, viz. $C_{\vec{a}, a^{\prime}}$. But then, $\mathcal{D}_{f}$ must already be principal, namely $\mathcal{D}_{f}=\{C \subseteq I: i \in C\}$ for some individual $i \in C_{\vec{a}, a^{\prime}}$. The individual $i$ is the dictator.

\footnotetext{
${ }^{9}$ Note (i) that we explicitly allow the case $m=0$, so that $\psi$ may be an existential statement, and (ii) that $\phi$ - which is assumed to be in normal form - might start with a block of existential quantifiers, in which case the first block of existential quantifiers in $\psi$ would consist of several quantifiers, not just one.

${ }^{0}$ Corrected after publication.
} 
2. By assumption, $f(\underline{\mathfrak{A}})$ is just the $A$-restriction of the reduced product of $\mathfrak{A}$ with respect to $\mathcal{D}_{f}$. Since $\phi$ is free of negation, disjunction and universal quantification, an analysis of the proof of Łoś's theorem reveals that we must have

$$
\begin{aligned}
& f(\underline{\mathfrak{A}}) \models \phi\left(\dot{a}_{1}, \ldots, \dot{a}_{m} ; \dot{a}^{\prime}\right) \\
\Leftrightarrow \quad & \left\{i \in I: \mathfrak{A}_{i} \models \phi\left(\dot{a}_{1}, \ldots, \dot{a}_{m} ; \dot{a}^{\prime}\right)\right\} \in \mathcal{D}_{f}
\end{aligned}
$$

for all $a_{1}, \ldots, a_{m}, a^{\prime} \in A$. Hence, as before one can show that the filter $\mathcal{D}_{f}$ contains a finite subset of $I$, viz. $C_{\vec{a}, a^{\prime}}$. But then, $\mathcal{D}_{f}=$ $\left\{C \subseteq I: C^{\prime} \subset C\right\}$ for some $C^{\prime} \subseteq C_{\vec{a}, a^{\prime}}$. This $C^{\prime}$, necessarily a finite set, is the set of oligarchs.

Already Lauwers and Van Liedekerke [10, p. 230, Property 4 (of aggregation functions)] had obtained a dictatorial impossibility theorem for preference aggregators that preserve certain non-universal formulae (i.e. formulae which contain at least one existential quantifier). However, their theorem is based on a syntactic condition which is quite restrictive as it entails that $A$ is countable and that $I$ is the set of nonnegative integers $\mathbb{N}$. (Lauwers and Van Liedekerke's [10] proof strategy consisted essentially in constructing an aggregator based on a free ultrafilter which does not preserve the truth value of the non-universal formula in question, because the element which satisfies it does, by construction, not belong to A.) Our condition allows uncountable sets of alternatives and uncountable populations.

Moreover, even in the special setting of countably many alternatives and individuals, our condition is at least as general as the one of Lauwers and Van Liedekerke [10]:

Theorem 4.2. Let $I=\mathbb{N}$ and $A=\left\{\alpha_{i}\right\}_{i \in \mathbb{N}}$. For all $n \in \mathbb{N}$, let $\psi_{n}$ be the formula

$$
\left.\left.\left.\ddot{(} \dot{\forall} \dot{x}_{1} \dot{)} \ldots \dot{(} \dot{\forall} \dot{x}_{m}\right) \ddot{(} \dot{\forall} \dot{y}\right) \ddot{(} \phi\left(\dot{x}_{1}, \ldots, \dot{x}_{m} ; \dot{\alpha}_{n+1}\right) \dot{\wedge} \dot{(} \phi\left(\dot{x}_{1}, \ldots, \dot{x}_{m} ; \dot{y}\right) \rightarrow \bigwedge_{j=0}^{n} \dot{y} \neq \dot{\alpha}_{j}\right) \dot{)} .
$$

If $T \cup\left\{\psi_{n}\right\}$ is consistent for all $n \in \mathbb{N}$, then there exists some $\underline{\mathfrak{A}} \in \Omega^{I}$ with finite witness multiplicity with respect to $\phi$.

Proof of Theorem 4.2. Suppose that $T \cup\left\{\psi_{n}\right\}$ is consistent for all $n \in \mathbb{N}$. Then there exists for every $n \in \mathbb{N}$ some model $\mathfrak{A}_{n}$ of $T \cup\left\{\psi_{n}\right\}$ with domain $A$ Then, for every $k \in \mathbb{N}$ and arbitrary $a_{1}, \ldots, a_{m} \in A$, the set

$$
\left\{n \in \mathbb{N}: \mathfrak{A}_{n} \models \phi\left[\dot{a}_{1}, \ldots, \dot{a}_{m} ; \dot{\alpha}_{k}\right]\right\}
$$

must contain $k-1$, but none of the integers $\geq k$. It is therefore finite. Since $A=\left\{\alpha_{k}\right\}_{k \in \mathbb{N}}$, we conclude that for all $a \in A$ and all $a_{1}, \ldots, a_{m} \in A$, the set

$$
\left\{n \in \mathbb{N}: \mathfrak{A}_{n} \models \phi\left[\dot{a}_{1}, \ldots, \dot{a}_{m} ; \dot{a}\right]\right\}
$$

\footnotetext{
${ }^{10}$ For, by completeness, there exists for every $n \in \mathbb{N}$ some model $\mathfrak{A}_{n}$ of $T \cup\left\{\psi_{n}\right\}$ with domain $A_{n}$, relational interpretations $R^{m} \subseteq A_{n}^{\delta(m)}(m \in \mathbb{N})$ and pairwise distinct constant interpretations $c_{a}^{n} \in A_{n}(a \in A)$. Since $T \cup\left\{\psi_{n}\right\}$ is universal, the restriction of this relational structure to $\left\{c_{n}^{a}: a \in A\right\}$ will still be a model of $T \cup\left\{\psi_{n}\right\}$. Without loss of generality, one may assume that $c_{n}^{a}=a$ for all $a \in A$.
} 
is finite. On the other hand, $\psi_{n}$ implies $\psi$, so each of the $\mathfrak{A}_{n}$ is a model of $\psi$. This proves that $\left\langle\mathfrak{A}_{n}\right\rangle_{n \in I}$ has finite witness multiplicity with respect to $\phi$.

Let us finally consider some applications of our impossibility theorem (Theorem 4.1):

- In preference aggregation, as already remarked by Lauwers and Van Liedekerke [10, p. 231], any Arrovian aggregator which preserves either continuity or the existence of upper bounds or lower bounds must be dictatorial.

- In propositional judgment aggregation à la Dietrich and List [3], this result means that a judgment aggregator which satisfies certain rationality axioms and preserves some existential conjunctive statement about the elements of the agenda must be oligarchic, provided usual agenda conditions are met and there exists a profile with finite witness multiplicity. Under stronger agenda conditions, we even have a dictatorial impossibility result for aggregators which preserve some non-universal statement.

- In modal propositional judgment aggregation, any rational aggregator which preserves some existential conjunctive statement about possible worlds (in the Kripke semantics) must be oligarchic, provided there exists a profile with finite witness multiplicity. Under stronger agenda conditions, we even have a dictatorial impossibility result for aggregators which preserve some non-universal statement about possible worlds.

\section{Conclusion}

In a model-theoretic framework for the analysis of aggregation problems the ultraproduct construction allows one to derive the correspondence between abstract aggregation rules in an Arrovian spirit and (ultra)filters of decisive coalitions on the set of individuals. Whilst this construction immediately reveals why dictatorship results do not carry over to the infinite case - where free ultrafilters exist - , it opens up another source of impossibility results, which we have analyzed in this paper: Non-universal statements are generically not preserved under aggregation. This problem is, of course, hardly surprising from the vantage point of model theory (given that an important use of ultraproducts is the enlargement of a given structure). However, it shows the strength of a seemingly innocuous condition on aggregation rules - viz. that the aggregate model has exactly the same domain as the individual models (the factor domains of the ultraproduct) - , as this requirement can only be met for sufficiently rich theories if the ultrafilter of decisive coalitions is principal, i.e. the aggregation rule is dictatorial.

\section{References}

[1] Bell, J., And Slomson, A. Models and ultraproducts. An introduction. North-Holland, Amsterdam, 1969.

[2] Campbell, D. Intergenerational social choice without the Pareto principle. Journal of Economic Theory 50, 2 (1990), 414-423. 
[3] Dietrich, F., And List, C. Arrow's theorem in judgment aggregation. Social Choice and Welfare 29, 1 (2007), 19-33.

[4] Dietrich, F., And List, C. Propositionwise judgment aggregation: the general case. mimeo, Nov 2009.

[5] Fishburn, P. Arrow's impossibility theorem: concise proof and infinite voters. Journal of Economic Theory 2, 1 (1970), 103-106.

[6] Grandi, U., And Endriss, U. First-order logic formalisation of Arrow's theorem. In Proceedings of the 2nd International Workshop on Logic, Rationality and Interaction (LORI-2009), vol. 5834 of Lecture Notes in Computer Science. Springer, Berlin, 2009, pp. 133-146.

[7] Herzberg, F., AND ECKert, D. Impossibility results for infiniteelectorate abstract aggregation rules. Journal of Philosophical Logic 41, 1 (2012), 273-286.

[8] Hodges, W. Building models by games, vol. 2 of London Mathematical Society Student Texts. Cambridge University Press, Cambridge, UK, 1985.

[9] Kirman, A., And Sondermann, D. Arrow's theorem, many agents, and invisible dictators. Journal of Economic Theory 5, 2 (1972), 267-277.

[10] LaUwers, L., ANd VAN Liedekerke, L. Ultraproducts and aggregation. Journal of Mathematical Economics 24, 3 (1995), 217-237.

[11] List, C., And Polak, B. Introduction to judgment aggregation. Journal of Economic Theory 145, 2 (2010), 441-466.

[12] List, C., AND Puppe, C. Judgment aggregation: A survey. In The Handbook of Rational and Social Choice: An Overview of New Foundations and Applications, P. Anand, P. Pattanaik, and C. Puppe, Eds. Oxford University Press, Oxford, 2009, pp. 457-482.

[13] Pauly, M., and van Hees, M. Logical constraints on judgement aggregation. Journal of Philosophical Logic 35, 6 (2006), 569-585.

[14] Rubinstein, A. The single profile analogues to multi profile theorems: Mathematical logic's approach. International Economic Review 25, 3 (1984), 719-730.

[15] Rubinstein, A., And Fishburn, P. Algebraic aggregation theory. Journal of Economic Theory 38, 1 (1986), 63-77.

[16] WiLson, R. On the theory of aggregation. Journal of Economic Theory 10, 1 (1975), 89-99. 